\title{
Le champ des sustainability transitions : origines, analyses et pratiques de recherche
}

\section{The field of sustainability transitions: origins, analysis and research practices \\ El campo de las transiciones sustentables: orígenes, análisis y prácticas de investigación}

\section{René Audet}

Numéro 58, hiver 2015

Pour une sociologie de la transition écologique

URI : https://id.erudit.org/iderudit/1036207ar

DOI : https://doi.org/10.7202/1036207ar

Aller au sommaire du numéro

Éditeur(s)

Athéna éditions

ISSN

0831-1048 (imprimé)

1923-5771 (numérique)

Découvrir la revue

Citer cet article

Audet, R. (2015). Le champ des sustainability transitions : origines, analyses et pratiques de recherche. Cahiers de recherche sociologique, (58), 73-93.

https://doi.org/10.7202/1036207ar

\section{Résumé de l'article}

Le champ des sustainability transitions est peu connu dans les cercles universitaires francophones, demeurant surtout, à ce jour, une affaire européenne. L'objectif de cet article est donc avant tout d'introduire ce champ au monde francophone : il s'agit de présenter les sustainability transitions, ses principales écoles, leurs problématiques théoriques et empiriques, leurs pratiques méthodologiques et leurs critiques. L'article est organisé autour des trois problématiques centrales du champ, soit : les problèmes de l'incertitude, de la complexité et de la réflexivité dans la gouvernance du développement durable, le fort degré de verrouillage des principaux systèmes sociotechniques empêchant leur " transition vers la durabilité ", et le défi plus méthodologique du pilotage du changement et des transitions. L'article présente ces trois problématiques en montrant leurs ancrages dans différentes filiations théoriques des sciences sociales et des sciences de l'environnement, et en propose finalement une critique épistémologique et politique qui invite à réfléchir davantage aux conséquences des sustainability transitions sur les transformations actuelles des sociétés dans le contexte des crises écologique et climatique. 


\section{Le champ des sustainability transitions: origines, analyses et pratiques de recherche}

RenÉ Audet

\section{Introduction}

Le champ des sustainability transitions est peu connu dans les cercles universitaires francophones. La controverse entourant la traduction française du terme sustainability y est peut-être pour quelque chose ${ }^{1}$, mais il faut remarquer que les chercheurs étatsuniens et canadiens-anglais n'ont pas davantage intégré ce champ. Ainsi, le champ des sustainability transitions demeure surtout, à ce jour, une affaire européenne : il a émergé à partir du début des années 2000 en Allemagne, au Royaume-Uni et aux Pays-Bas avant de se diffuser inégalement ailleurs dans le monde. Il s'est développé à travers un processus d'échange entre trois écoles de pensée issues de ces pays qui, très tôt, a donné lieu à une certaine organisation du champ. Ainsi, depuis 2010, il est doté d'un réseau international (le Sustainability Transitions Research Network) qui tient une conférence annuelle (International Sustainability Transitions Conference) et publie une revue scientifique (Environnemental Innovation and Societal Transitions).

1. Voir Edwin Zaccaï, Le développement durable. Dynamique et constitution d'un projet, Bruxelles, P...E. Peter Lang, 2005; Corinne Gendron, Vous avez dit développement durable?, Montréal, Presses internationales Polytechnique, 2007. 
Les publications francophones sur le sujet sont donc rares. La plus importante est sans doute l'article de Boulanger ${ }^{2}$, un chercheur belge qui présente deux des trois courants des sustainability transitions dans une perspective avant tout informative. Les travaux plus empiriques fondés sur ces approches sont quasi inexistants en langue française, bien que l'auteur de ces lignes soit responsable de quelques tentatives ${ }^{3}$ et que l'article de Claire Lamine dans le présent numéro témoigne de sa diffusion croissante et de l'intérêt grandissant que suscitent les sustainability transitions. Dans ce contexte, l'objectif de cet article est avant tout d'introduire ce champ au monde francophone: il s'agit de présenter les sustainability transitions, ses principales écoles, leurs problématiques théoriques et empiriques, leurs pratiques méthodologiques et leurs critiques. Ainsi, j'espère surtout donner un aperçu de l'intérêt et des limites que le champ des sustainability transitions présente pour l'analyse des transformations sociales et techniques en contexte de crise écologique et climatique.

Du point de vue scientifique, ce champ s'organise autour de trois problématiques centrales. La première est celle de l'incertitude dans laquelle l'action et la gouvernance en faveur de la «durabilité» sont plongées aujourd'hui. Le thème de la réflexivité se pose alors comme une réponse éventuelle et ouverte aux défis de l'incertitude. La deuxième problématique constate le fort degré de verrouillage des principaux systèmes sociotechniques et s'interroge sur les facteurs capables de radicalement transformer ces systèmes, notamment l'innovation sociotechnique. Réflexivité et innovation se conjuguent en quelque sorte au point de départ de la troisième problématique - de nature plus méthodologique - qui est celle du «pilotage» du changement et de l'intervention scientifique dans la transformation du monde. C'est dans cet ordre que j'aborde les trois écoles des sustainability transitions dans cet article. Cette présentation est précédée d'une première section explorant les filiations théoriques communes à ces trois écoles, et suivie d'une dernière section portant sur les principales critiques formulées à leur endroit.

\section{Filiations théoriques}

La «transition» se pose aujourd'hui comme un objet de discours ralliant une multitude d'acteurs et de pratiques associées en matière de lutte contre la crise écologique ${ }^{4}$. Le terme n'est cependant pas nouveau dans le discours

2. Paul-Marie Boulanger, «Une gouvernance du changement sociétal: le transition management», Revue Nouvelle, novembre 2008, p. 61-73.

3. Voir notamment Sylvain Lefèvre et René Audet, «La mise en marché alternative de l'alimentation à Montréal. De la niche d'innovation à une transition du secteur alimentaire?», Interventions économiques, n 54, 2016.

4. René Audet, «Discours autour de la transition», dans Marie-José Fortin, Yann Fournis et François L'Italien (dir.), La transition énergétique. Les configurations institutionnelles et territoriales de l'énergie, Québec, Les Presses de I'Université Laval, 2016, p. 11-30 
environnemental. Il apparaissait déjà dans l'un des textes fondateurs des sciences de l'environnement. En effet, le Rapport Meadows sur Les limites de la croissance envisageait dès 1972 une «transition vers l'équilibre global» supportée par une gouvernance mondiale de l'environnement davantage centralisée et rationnelle, plus apte à affronter les défis globaux de la surpopulation, de la pollution galopante et de la finitude des ressources naturelles ${ }^{5}$. Bien que le projet d'une organisation mondiale de l'environnement ne se concrétisa jamais, l'idée même d'une rationalisation croissante des politiques publiques en matière d'environnement était dans l'ère du temps - la décennie 1970 fut d'ailleurs celle de la création des ministères de l'Environnement dans la plupart des pays occidentaux. Dès lors, l'action publique se déploya dans ce nouveau domaine en appliquant les préceptes de la rationalisation, notamment qu'il est possible de cerner un "problème» et d'y trouver une solution en contrôlant par la réglementation les flux à son origine.

Ainsi, il s'agissait moins d'une véritable transformation structurelle que de l'inclusion d'un nouveau champ dans le processus de modernisation - d'où l'expression «modernisation écologique» qui occupa une large partie du débat universitaire en sociologie de l'environnement jusqu'au début des années $2000^{6}$. La modernisation écologique demeure une notion large et polysémique. On peut la définir comme:

[...] le discours qui reconnait le caractère structurel de la problématique environnementale mais qui assume néanmoins que les institutions politiques, économiques et sociales existantes peuvent internaliser la préoccupation environnementale. Pour ce faire, la modernisation écologique introduit des concepts qui, avant tout, rendent les dégradations environnementales calculables ${ }^{7}$.

L'opérationnalisation de la modernisation écologique dans les politiques environnementales s'est effectuée à travers la promotion de technologies moins polluantes, d'une gouvernance plus participative, de mécanismes de marché comme les certifications et autres processus de normalisation des activités industrielles ainsi que par la réglementation des émissions pour un grand nombre de polluants et de sous-produits industriels. Ces normes d'émission, en particulier, constituent des formes de légalisation de la pollution encadrée par un processus discursif de définition dominé par les sciences naturelles et techniques qui décident, sur la base d'analyses en laboratoire et

5. Donella H. Meadows, Dennis L. Meadows, Jorgen Randers et William W. Behrens, The Limites to Growth, New York, Universe Books, 1972.

6. Arthur Mol, «Ecological Modernisation Theory in Debate: A Review», Environmental Politics, vol. 9, n 1, 2000, p. 92-115.

7. Marteen A. Hajer, The Politics of Environmental Discourse. Ecological Modernization and the Policy Process, Oxford, Oxford University Press, 1995, p. 25-26. Sauf indication contraire, toutes les traductions sont de l'auteur. 
d'études de faisabilité, ce qui est tolérable pour la santé des écosystèmes et la santé humaine.

Scientifiquement, affirme Beck, l'aspect rationnel des taux limites n'est qu'apparence : ceux-ci ne tiennent pas compte des effets de diffusion hétérogène dans l'environnement, de l'interaction avec d'autres contaminants ou des différents phénomènes d'exposition dans la société. Ainsi, l'expérimentation sur les taux limites ne se fait pas dans les laboratoires, mais dans le monde directement, ce qui fait dire à Beck que «la science s'est transformée en admi-

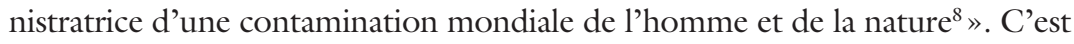
lorsque cette contamination devient apparente et que le risque devient objet de construction sociale et de conflit que l'on entre dans une "société du risque", laquelle ébranle les fondements même de la modernisation écologique et de ses préceptes rationalistes:

La société du risque renvoie précisément à une constellation dans laquelle l'idée de contrôlabilité fondée sur des décisions concernant les effets collatéraux et les dangers - idée qui est un principe directeur de la modernité - est devenue questionnable. Il s'agit d'une constellation dans laquelle un nouveau savoir sert à transformer des risques imprévisibles en risques calculables, mais qui par ailleurs donne lieu à de nouvelles imprévisibilités qui nous forcent à réfléchir sur les risques $[\ldots]^{9}$.

Voilà en quoi la société du risque est nécessairement réflexive : elle contraint à penser le changement social à partir des conséquences imprévues et non volontaires des actions humaines. Les analyses de Beck sur la société du risque ont largement contribué à mettre en cause la prétention rationaliste de la modernisation écologique et à inspirer l'émergence du champ des sustainability transitions, notamment en introduisant l'idée d'une gouvernance «réflexive» intrinsèquement non linéaire.

Mais ces analyses ne furent pas les seules à alimenter les sustainability transitions. Au même moment, en sciences de l'environnement et surtout en écologie, l'émergence du paradigme de la complexité interrogeait la pertinence des relations linéaires de cause à effet dans les dynamiques écosystémiques. Ces sciences, qui reposaient largement sur une théorie des systèmes fonctionnaliste et formaliste issue de la thermodynamique ${ }^{10}$, intégrèrent bientôt la mathématique du chaos, avec ses concepts corollaires "d'attracteur", «d'équilibre spontanée» et de "points de rupture ${ }^{11}$. La nouvelle théorie des systèmes complexes, plus probabiliste, continue néanmoins de prétendre

8. Ulrich Beck, La société du risque, Paris, Alto Aubier, 2001, p. 127.

9. Ulrich Beck, World at Risk, Cambridge, Polity Press, 2009, p. 15.

10. Voir Jean-Paul Deléage, Histoire de l'écologie une science de l'homme et de la nature, Paris, La Découverte, 1991.

11. Voir Simon A. Levin, «The Problem of Patterns and Scale in Ecology: The Robert H. MacArthur Award Lecture», Ecology, vol. 73, n 6, 1992, p. 1943-1967. 
- grâce notamment aux avancées des outils de modélisation - à une description intégrée de plusieurs «niveaux» d'organisation de la matière et du vivant, allant du système climatique et de la biosphère aux écosystèmes plus locaux. Elle passe alors d'une théorie de l'ordre qui décrivait des flux dans une perspective d'équilibre et de «climax» à une théorie du changement qui porte sur la «résilience» des systèmes et leur «capacité d'adaptation» ${ }^{12}$. Ici, les «propriétés émergentes» des systèmes - il s'agit de nouvelles fonctions qui font basculer un système vers un autre équilibre dynamique - donnent lieu à une complexité croissante qui culmine dans les «systèmes socio-écologiques». Ainsi, il n'y a qu'un pas à faire pour en arriver à une application de la théorie des systèmes complexes aux sociétés humaines - un pas que certains auteurs des sustainability transitions n'hésitent pas à faire: «l'hypothèse sous-jacente de la théorie de la transition est que la société change de manière plutôt évolutionnaire et organique, comparable jusqu'à un certain point au comportement et au développement des écosystèmes ${ }^{13}{ }^{1}$.

Les concepts de réflexivité et de complexité sont au cour des théories de la transition et du champ des sustainability transitions, bien que ceux-ci soient différemment interprétés, hiérarchisés et associés à d'autres concepts au sein des trois approches que sont: la gouvernance réflexive, la perspective multiniveaux des transitions sociotechniques et la gestion des transitions. Je les aborde maintenant dans cet ordre.

\section{La gouvernance réflexive des transitions}

Les approches technologiques, de politique publique ou de gestion organisationnelle tendent à poser les problèmes en isolant un phénomène ou une variable d'une réalité complexe pour en comprendre les causes et les effets, afin d'agir en conséquence. Or, les problèmes environnementaux répondent mal à cette logique rationaliste. Leur complexité, comme l'affirment les tenants de la gouvernance réflexive, les rend "vilains» (wicked) ou ambigus. Ces problèmes sont

si complexes parce qu'ils sont liés à des enjeux économiques et sociaux aussi bien que spatiaux et écologiques. Les problèmes se manifestent à différents niveaux, impliquent de nombreux acteurs (et non seulement des acteurs corporatifs) et requièrent beaucoup de temps pour être géré efficacement ${ }^{14}$.

12. Par exemples: Carl Folke, «Resilience: The emergence of a perspective for social-ecological systems analyses», Global Environmental Change, vol. 16, 2006, p. 253-267; Gilberto C. Gallopin, «Linkages between vulnerability, resilience, and adaptive capacity», Global Environmental Change, vol. 16, 2006, p. 293-303.

13. René Kemp et Derk Loorbach, «Transition management: a réflexive governance approach », dans Jan-Peter Voß et al. (dir.), Reflexive Governance for Sustainable Development, Cheltenham/Northamption, Edward Elgar, 2006, p. 103.

14. Ibid., p. 104. 
C'est pourquoi il convient de penser le changement social en tenant compte de la complexité, de l'incertitude et de l'ambigüité des problèmes de durabilité. Leur gouvernance réflexive nécessite d'interrompre les automatismes et les routines de la gestion rationaliste en portant le regard sur les conditions et les effets de cette gestion rationaliste ${ }^{15}$.

L'entreprise qui consiste à concevoir les problèmes de durabilité sous l'angle de la complexité est ambitieuse, mais elle ne demeure qu'un préalable au véritable projet de la gouvernance réflexive qui est d'orienter le changement social vers un état plus durable. Ainsi, il est hors de question d'attendre que les structures se transforment sous leurs propres dynamiques, ni que l'action héroïque d'un groupe d'acteur n'y apporte une solution définitive. La gouvernance réflexive mise plutôt sur des formes de gestion du changement inspirées de ce que Giddens appelle la «dualité du structurel ", c'est-à-dire le processus récursif qui préside à la fois à la reproduction des structures sociales et à leur transformation en «habilitant» les acteurs sociaux à innover dans certaines limites ${ }^{16}$. Ainsi le sociologue John Grin affirme qu'il existe une occasion de transformer les pratiques récursives à travers la formation de la conscience discursive des problèmes de durabilité ${ }^{17}$. Pour lui, la «re-structuration » nécessaire pour affronter la crise écologique renvoie aux transformations interreliées de la structure et de l'action à travers des processus délibérés et guidés. Dans le même sens, ses collègues Voß et Kemp affirment que :

La gouvernance réflexive renvoie au problème du façonnage du développement social à la lumière de la réflexivité des stratégies de conduite - le fait que la réflexion et l'action concernant un objet de conduite affectent aussi le sujet et son habilité à conduire ${ }^{18}$.

Le terme «pilotage» utilisé dans le courant des sustainability transitions renvoie donc à des approches procédurales de gestion du changement visant à engendrer une telle re-structuration - ou «transition». Le terme transition, ici, rend davantage visible l'articulation entre le projet de gouvernance réflexive et la théorie des systèmes complexes - son sens va au-delà de la définition générique (le passage d'un état vers un autre) et acquiert une signification substantive spécifique: le passage d'un état d'un système vers un autre état du même système:

Une transition est le résultat de l'interaction de développements qui se supportent et se renforcent les uns les autres. Les transitions ne sont pas causées par des

15. Jan-Peter Voß et René Kemp, «Sustainabilty and Reflexive Governance: Introduction », dans Jan-Peter Voß et al. (dir.), op. cit.

16. Anthony Giddens, La constitution de la société. Éléments de la théorie de la structuration, Paris, Presses Universitaires de France, 1987.

17. John Grin, «Reflexive Modernisation as a Governance Issue, or: designing and Shaping Re-structuration », dans Jan-Peter Voß et al. (dir.), op. cit., p. 57-81.

18. Jan-Peter Voß et René Kemp, op. cit., p. 4. 
variables singulières - la variation des prix, une politique ou une nouvelle technologie - mais par les résultats de développements dans de multiples domaines qui s'alimentent entre eux : la technologie, l'économie, les institutions, les comportements, la culture, l'écologie et les paradigmes ${ }^{19}$.

Dans ce contexte, le pilotage se définit plus précisément comme «une tentative intentionnelle pour emmener un système d'un état vers un autre en exerçant de l'influence sur ses dynamiques de développement ${ }^{20}{ }$. Or, étant donné l'ambigüité et la complexité des problèmes de durabilité, et notamment leur dimension socialement construite, tout exercice de pilotage doit tenir compte des objectifs vagues et conflictuels des acteurs sociaux, de l'incertitude des connaissances et de la distribution hétérogène du pouvoir dans la société du risque. C'est pourquoi la formation de la conscience discursive et la pratique de la réflexivité apparaissent si nécessaire - piloter le changement, c'est «améliorer la réflexivité des acteurs à l'égard de l'interdépendance et l'imbrication dans des contextes systémiques, ainsi que faciliter l'adaptation mutuelle au sein de processus continus de développement sociétal ${ }^{21}{ }^{2}$.

Dans la pratique, le pilotage du changement consiste à créer des espaces permettant d'engendrer cette réflexivité, à réunir des acteurs pour organiser une communication et une interaction orientée vers une compréhension commune et une résolution coordonnée des problèmes ${ }^{22}$. Ces processus organisés de pilotage mettent notamment en ouvre des méthodes de prospective qui permettent d'élaborer collectivement des images mentales de futurs possibles et de guider en conséquence les actions, les acteurs et leurs interactions. C'est, du moins, ainsi que fonctionne le modèle le mieux documenté dans la littérature des sustainability transitions, la «gestion des transitions» (transition management), présenté plus bas.

Sur la base d'une remise en question de l'approche rationaliste et linéaire de la résolution des problèmes de durabilité, les tenants de la gouvernance réflexive se proposent donc de développer des méthodes permettant de piloter le changement sur la base d'objectifs socialement construits et négociés. Bien que l'argumentaire donne lieu à un projet davantage méthodologique, il faut convenir que le propos de la gouvernance réflexive demeure relativement abstrait. Peu d'attention est réellement portée aux multiples domaines ou structures qui interagissent dans les processus de transition. Le pilotage

19. René Kemp et Derk Loorbach, op. cit., p. 106.

20. Jan-Peter Voß, Jens Newig, Brita Kastens, Jochen Monstadt et Benjamin Nölting, «Steering for Sustainable Development: A Typology of Problems and Strategies with respect to Ambivalence, Uncertainty and Distributed Power », dans Jens Newig et al. (dir.), Governance for Sustainable Development. Coping with Ambivalence, Uncertainty and Distributed Power, Londres/New York, Routledge, 2008, p. 3.

21. Ibid., p. 12.

22. Jan-Peter Voß et René Kemp, op. cit. 
du changement est posé théoriquement dans les termes de la réflexivité et de la complexité, mais le contexte sociologique concret dans lequel ce pilotage doit avoir lieu n'est pas très bien défini. Le deuxième courant des sustainability transitions porte précisément sur ce contexte sociologique et fournit une toile de fond analytique dont les méthodes de pilotage du changement peuvent s'inspirer pour envisager «l'état d'un système » qu'il s'agit de transformer.

\section{La perspective multiniveaux sur les transitions sociotechniques}

Les trois courants des sustainability transitions partagent l'idée qu'une transition procède toujours par transformations multiples, simultanées, interférant mutuellement et agissant à des niveaux variés allant du plus local au plus structurel et macrosociologique. Mais parmi ces trois courants, la perspective multiniveaux est l'approche qui décrit le mieux la nature et la dynamique de ces transformations. Ses filiations théoriques ne sont pas étrangères aux thèses de la modernité réflexive et de la complexité : la première étant réinterprétée dans le cadre de la théorie de la structuration de Giddens et la seconde étant retraduite dans le langage de l'économie évolutionniste ${ }^{23}$. Ce qui distingue toutefois plus nettement la perspective multiniveaux des deux autres approches, sur le plan des filiations théoriques et disciplinaires, est son ancrage dans le champ des études de l'innovation. À cet égard, la perspective multiniveaux participe à un recadrage de l'analyse de l'innovation: aux considérations purement techniques ou économiques du succès et de l'échec des innovations, celle-ci oppose l'idée de «régimes sociotechniques» au sein desquels les innovations émergent, évoluent, s'imposent ou disparaissent ${ }^{24}$.

L'objet de la perspective multiniveaux est donc le régime sociotechnique davantage que l'innovation elle-même. La multiplicité des niveaux dans les régimes sociotechniques suppose une certaine verticalité entre des échelles de structuration qui n'est pas sans rappeler la «dualité du structurel» que pose Giddens dans sa théorie de la structuration: un domaine de l'agent qui renvoie à l'activité continue des individus dans le cadre de la conscience pratique et de la conscience discursive, et le domaine des structures, qui sont des «ensembles de règles et de ressources engagés de façon récursive dans la reproduction des systèmes sociaux $»^{25}$. Pour Giddens, la structuration implique un phénomène de récursivité entre agents et structures qui assure la

23. Frank G. Geels et Johan Schot, «The Dynamics of Transitions. A Socio-Technical Perspective», dans John Grin, Jan Rotmans et Johan Schot (dir.), Transitions to Sustainable Development. New Directions in the Study of Lon Term Transformational Change, New York, Londres, Routledge, 2010, p. 9-101.

24. Adrian Smith, Jan-Peter Voß et John Grin, «Innovation studies and sustainability transitions: The allure of the multi-level perspective and its challenges», Research Policy, vol. 39, 2010, p. 435-448.

25. Anthony Giddens, op. cit., p. 444. 
reproduction de celles-ci tout en habilitant l'action de ceux-là. La perspective multiniveaux admet d'emblée cette dualité du structurel et cherche à l'adapter au contexte des régimes sociotechniques. Son projet consiste à illustrer l'existence de niveaux plus structurés et stables qui imposent une pression de «sélection» aux innovations, et d'espaces plus «habilitants» où l'innovation dite «radicale» demeure possible. Il s'agit donc d'examiner les interactions «verticales» entre les niveaux et de découvrir, pour un régime sociotechnique donné, les principes qui participent à sa reproduction et les forces qui pourraient réussir à le transformer dans le sens d'une plus grande durabilitée ${ }^{26}$.

Ainsi, la perspective multiniveaux propose trois niveaux soumis à divers degrés au poids des structures et à des temporalités de transformation distinctes: le régime sociotechnique est le niveau central, car c'est lui qui fera l'objet de transitions sous la pression venant d'un niveau supérieur, le "paysage sociotechnique» et d'un niveau inférieur, la "niche d'innovation radicale». Avant d'en faire une présentation plus détaillée, précisons que ces trois niveaux s'appliquent à des grands domaines d'activité des sociétés humaines - il ne s'agit pas de penser «la société » à travers cette lentille, mais plutôt d'examiner des secteurs comme la mobilité, l'agriculture, la production et la distribution énergétique, l'aménagement urbain, etc.

Il convient d'aborder en premier le niveau du régime sociotechnique luimême, qui est au cour de la perspective multiniveaux et qui est celui qui fera l'objet de «transitions vers la durabilité». Un régime sociotechnique est une configuration d'acteurs, de technologies, d'infrastructures et de ressources dont le fonctionnement est organisé par des règles et des pratiques institutionnalisées. Les acteurs et organisations impliqués dans un régime sociotechnique sont nombreux et diversifiés: instances gouvernementales, centres de recherches et de développement, entreprises et associations industrielles, corporations professionnelles, etc. Ces acteurs forment des réseaux de relations autour de technologies et d'infrastructures matérielles qui sont relativement stables, ou «verrouillés» comme le disent les penseurs de la perspective multiniveaux. Cette stabilité témoigne en fait de la reproduction des réseaux de relations au travers d'un ensemble de règles qui sont de trois types ${ }^{27}$.

Premièrement, les « règles cognitives» renvoient aux systèmes de croyance et de représentations qui fondent les manières de faire dans les domaines techniques. Ainsi, la recherche et développement est régie par des routines institutionnalisées auxquelles correspondent des rôles bien définis pour les ingénieurs, les chercheurs, les aides gouvernementales ou les entrepreneurs.

26. Frank G. Geels et Johan Schot, op. cit., p. 18-19.

27. Frank W. Geels, «From sectoral systems of innovation to socio-technical systems. Insights about dynamics and change from sociology and institutional theory», Research Policy, vol. 33, 2004, p. 897-920. 
Deuxièmement, les «règles de régulation» sont des dispositifs conventionnels émanant du droit - réglementations environnementales fondées sur l'imposition des taux limites, droit commercial et civil qui fondent les règles entourant la propriété intellectuelle, etc. - ou de réseaux hybrides de normalisation comme l'Organisation internationale de normalisation ou le Conseil canadien des normes. Troisièmement, les règles normatives portent sur les valeurs partagées et les définitions socialement construites de la qualité, de l'efficacité, de la durabilité, etc.

Les théoriciens de la perspective multiniveaux affirment que «les règles des régimes sociotechniques expliquent la stabilité et le verrouillage des systèmes sociotechniques ${ }^{28}$ ». C'est d'ailleurs tout le défi de ceux qui voudraient transformer ces régimes: ils sont si profondément verrouillés que seules les innovations incrémentales semblent pouvoir transformer les régimes sociotechniques. Et encore, ces transformations incrémentales, même cumulées, ne sont pas de nature à transformer la structure et les règles du régime. C'est pourquoi une transition vers la durabilité nécessite des interventions externes, provenant des deux autres niveaux, lesquelles obéissent à des logiques et à des temporalités différentes ${ }^{29}$.

Le niveau supérieur de la perspective multiniveaux est le "paysage sociotechnique ». Si l'on peut dire que les régimes sociotechniques évoluent dans une temporalité qui est conjoncturelle (des transitions peuvent se produire par intervalle de quelques décennies), les paysages sociotechniques se transforment plus lentement, à une rythme de "longue durée » comme le disait l'historien Fernand Braudel ${ }^{30}$. Ces paysages constituent l'environnement exogène des régimes et notamment les manifestations matérielles de l'organisation sociale et les biotopes dans lesquels évoluent les régimes sociotechniques. D'autres processus sociohistoriques sont inclus dans la notion de paysage sociotechnique, comme la mondialisation ou l'industrialisation, des chocs ponctuels comme les guerres, les catastrophes écologiques, les crises boursières: autant d'événements qui peuvent théoriquement fragiliser la stabilité des régimes sociotechniques. Puisque, dans la réalité, tous les régimes sociotechniques sont interreliés de multiples façons, un régime sociotechnique donné peut affecter le paysage d'un autre régime sociotechnique le prix de l'énergie, par exemple, est de nature à influencer la plupart des régimes.

28. Frank G. Geels et Johan Schot, op. cit., p. 20.

29. Ibid.

30. Fernand Braudel, «Histoire et sciences sociales: la longue durée», Annales, vol. 13, 1958, p. 725-753. 
Ainsi, de l'aveu même de ses théoriciens, le niveau du paysage sociotechnique est le plus difficile à définir ${ }^{31}$, et il convient peut-être mieux de le considérer comme l'ensemble des forces structurelles qui peuvent exercer des pressions à la transformation des régimes sociotechniques. Dans la perspective multiniveaux, ces pressions ne sont pas porteuses de solutions spécifiques et ne permettent pas de prédire la direction du changement. Toutefois, les auteurs considèrent qu'elles peuvent ouvrir des «fenêtres d'opportunité » pour l'intégration dans les régimes sociotechniques de nouveaux réseaux d'acteurs, de technologie et de règles qui étaient auparavant considérés trop radicaux.

Ces réseaux d'innovation plus radicaux prennent naissance dans le niveau inférieur de la perspective multiniveaux, celui des «niches d'innovation radicale». Il s'agit d'espaces de relative autonomie, évoluant en marge des régimes, et qui bénéficient d'une certaine protection à l'égard des "pressions de sélection» qui émanent de la domination des régimes sociotechniques dans leurs secteurs d'activité. Ainsi, les innovations de niches sont générées par des acteurs porteurs de normativités et de solutions alternatives incompatibles avec les règles des régimes - il peut s'agir de projets expérimentaux, d'innovations techniques ou sociales à petite échelle. Étant donné leurs relative incompatibilité avec les règles des régimes verrouillés, leur rythme d'apparition et de disparition est rapide - il correspond à une temporalité plus ponctuelle. Mais bien que leur destin soit très souvent la disparition pure et simple, certaines innovations réussissent à se consolider à l'abri des pressions de sélection, notamment parce que des acteurs choisissent d'y investir des ressources et de les "protéger» de ces pressions ${ }^{32}$. C'est par exemple la fonction des «Fab lab» et autres incubateurs d'innovation qui se multiplient aujourd'hui un peu partout dans le monde.

Dans certaines circonstances, lorsque la conjoncture est adéquate - par exemple lorsqu'un choc provenant du paysage sociotechnique ouvre des «fenêtres d'opportunité» - et qu'une niche d'innovation a atteint un degré de structuration suffisant, il est possible de voir des innovations s'imposer au niveau du régime, que ce soit de manière harmonieuse ou non. Si ce processus a pour effet de déverrouiller les réseaux d'acteurs, de technologies et de règles du régime sociotechnique, alors on peut parler d'une transition. En fait, les théoriciens de la perspective multiniveaux conçoivent de nombreuses

31. Frank W. Geels, «The multi-level perspective on sustainability transitions: Responses to seven criticisms», Environmental Innovation and Societal Transitions, vol. 1, n 1, 2011, p. 24-40.

32. Frank G. Geels et Johan Schot, op. cit., p. 22. 
«trajectoires» pouvant mener à des transitions ${ }^{33}$. Sur la base de l'analyse de cas historiques - la transition du bateau à voile au bateau à vapeur, la transition vers le système d'égout dans les villes européennes, la transition de la mobilité à cheval à la voiture dans les villes américaines et la transition de la manufacture conventionnelle à la production de masse aux États-Unis ceux-ci élaborent une typologie de trajectoires qui impliquent divers degrés de verrouillages des régimes, divers types de pression provenant du paysage et divers niveaux de structuration des niches d'innovation radicale. Une transition n'est donc jamais un phénomène linaire - c'est toujours un processus multiniveaux, multiacteurs, multiphase et coévolutif ${ }^{34}$.

Stratégiquement, du point de vue de la niche d'innovation radicale, on peut donc poser l'axiome suivant : la clé du succès et le meilleur levier concret pour engendrer une transition vers la durabilité reposent sur la capacité des niches à générer et à intégrer de manière réflexive des réseaux d'acteurs et d'innovation qui se renforcent mutuellement en créant et reproduisant des règles à travers leurs actions, expérimentations, projets et pratiques. Il s'agit en quelque sorte, comme le dirait Giddens, de créer une conscience pratique et une conscience discursive au sein des niches d'innovation radicale.

On arrive enfin, en combinant les prescriptions de la gouvernance réflexive et de la perspective multi-niveaux, à un arrière-plan théorique plus complet pour élaborer une méthode de pilotage du changement. C'est là la fonction, au sein des sustainability transitions, du courant de transition management.

\section{La gestion des transitions}

Les prescriptions de la gouvernance réflexive concernant le pilotage du changement et les conclusions de la perspective multiniveaux sur la gestion stratégique des niches s'alignent toutes vers une certaine intervention des chercheurs dans des processus de changement social. C'est peut-être pourquoi elles s'accommodent bien avec la méthode de la gestion des transitions élaborée par des chercheurs néerlandais depuis les années 1990. Cette méthode ne s'est pas seulement développée dans le cadre universitaire - elle est aussi le résultat d'une collaboration des chercheurs avec le gouvernement néerlandais dès le début des années 2000, alors que ce dernier préparait son Plan

33. Frank W. Geels et Johan Schot, «Typology of sociotechnical transition pathways», Research Policy, vol. 36, 2007, p. 399-417; Frank W. Geels et René Kemp, «Dynamics in socio-technical systems: Typology of change processes and contrasting case studies», Technology and Society, vol. 29, 2007, p. 441-455.

34. John Grin, Jan Rotmans et Johan Schot, «Introduction. From Persistent Problems to System Innovations and Transitions», dans John Grin, Jan Rotmans et Johan Schot (dir.), op. cit., p. 1-8. 
national de politique environnementale ${ }^{35}$. Ainsi, plusieurs projets de gestion des transitions ont trouvé leur origine dans les objectifs, priorités et financements mis de l'avant dans ce plan gouvernemental ${ }^{36}$.

L'approche de la gestion des transitions représente donc, au sein des sustainability transitions, l'approche la plus appliquée:

Il y a au sein du champ de la recherche sur les transitions une quantité significative de recherche fondamentale, mais la recherche liée à la gestion des transitions est par définition (partiellement) appliquée et participative. Alors que plusieurs disciplines scientifiques (comme la science politique) sont timides face aux approches normatives, la recherche sur les transitions rendent explicites leurs ambitions $[\ldots]$ et, de là, pose le besoin de développer des méthodologies qui assureront que le processus de recherche lui-même est aussi structuré et transparent que possible ${ }^{37}$.

L'idée centrale de la gestion de la transition est d'organiser - dans des contextes définis et territorialement circonscrits - les processus de changement de manière réflexive. Kemp et Loorbach la décrivent comme «tournée vers l'avenir, adaptative, multiacteur, visant la gouvernance de processus de transformation à long terme offrant des bénéfices en termes de durabilité ${ }^{38}$ ». En intégrant cette approche aux politiques publiques, il s'agirait d'en arriver à une «adaptation réflexive» des institutions et des régimes, dans une perspective de soutenabilité et de bien commun. Toutefois, depuis les premiers projets en collaboration avec le gouvernement néerlandais, la gestion de la transition a surtout été mise en œuvre dans des contextes plus locaux, à plus petite échelle aux Pays-Bas et dans d'autres pays européens ${ }^{39}$.

La gestion de la transition propose d'organiser des «expériences» de transition à travers une série de principes et d'outils «construits autour du paradoxe que le changement social est trop complexe à manipuler en termes de gestion [mais que] l'acquisition de connaissance sur la complexité sociale - en adoptant une approche des systèmes complexes - peut aider à sonder les possibilités pour influencer la complexité sociale ${ }^{40} »$. Trois de ces prin-

35. Adrian Smith et Florian Kern, «The transitions storyline in Dutch environmental policy», Environmental Politics, vol. $18, n^{\circ} 1,2009$, p. 78-98.

36. Derk Loorbach et Jan Rotmans, «The practice of transition management: Examples and lessons from four distinct cases», Futures, vol. 42, 2010, p. 237-246; John Grin, Jan Rotmans et Johan Schot, « On patterns and agency in transition dynamics: Some key insights from the KSI programme», Environmental Innovation and Societal Transitions, vol. 1, 2011, p. 76-81.

37. Derk Loorbach, Transition Management. New Mode of Governance for Sustainable Development, Utrecht, International Books, 2007, p. 37.

38. René Kemp et Derk Loorbach, op. cit., p. 103.

39. MUSIC, 2014. The MUSIC Project. En ligne: www.themusicproject.eu/, page consultée le 31 mars 2014; Julia Maria Wittmayer, Niko Schäpke et Frank van Steenbergen, « Making sense of sustainability transitions locally: how action research contributes to addressing societal challenges», Critical Policy Studies, vol. 8, n 4, 2014, p. 465-485.

40. Jan Rotmans et Derk Loorbach, «Complexity and Transition Management», Journal of Industrial Ecology, vol. 13, $n^{\circ} 2,2009$, p. 188. 
cipes - tous largement issus de la pensée systémique, mais aussi révélés par la perspective multiniveaux - apparaissent plus centraux afin de comprendre le processus de gestion des transitions: les transitions procèdent en plusieurs phases, à plusieurs niveaux, et selon plusieurs patterns.

Premièrement, les transitions se déroulent à travers une succession non linéaire de phases et de dynamiques relativement rapides ou lentes. Selon les théoriciens de la gestion des transitions, la capacité de distinguer entre ces phases peut guider l'intervention en fournissant une vision analytique de l'état d'un système spécifique ou d'une niche d'innovation donnée. Deuxièmement, comme le propose la perspective multiniveaux, les transitions se produisent à l'intersection de plusieurs niveaux de structuration. Troisièmement, les transitions procèdent de mécanismes de changement communs aux systèmes complexes que sont la variation, la sélection, l'adaptation, l'émergence, etc., et qui se matérialisent dans les interactions entre les différents niveaux que sont le paysage, le régime et la niche ${ }^{41}$. Ainsi, à l'approche plus sociologique des théoriciens de la perspective multiniveaux, la gestion des transitions ajoute des catégories théoriques directement tirées de la théorie des systèmes complexes.

C'est la synthèse des trois principes énumérés ci-haut qui donne lieu à un «modèle opérationnel de mise en ouvre: le cycle de la gestion de la transition $^{42} »$. Ce cycle comprend quatre phases distinctes, toutes animées et supervisées par une équipe de chercheurs; ce sont les phases stratégique, tactique, opérationnelle et réflexive. Ainsi, le cycle de la gestion de la transition fonctionne-t-il en suivant des étapes distinctes nécessitant chaque fois des compétences différentes, et en intégrant de nouveaux acteurs à chaque phase. Généralement, une initiative de gestion de la transition émerge de l'intérêt d'une équipe de chercheur envers un problème de durabilité géographiquement situé, de l'ouverture des autorités publiques pour s'engager dans le processus (et parfois de le soutenir financièrement), et de la volonté des acteurs locaux d'y prendre part activement. Une fois ces conditions réunies, le cycle démarre avec la constitution d'une «arène de transition» qui supervisera l'ensemble des travaux.

En fait, la constitution de cette arène de transition est le cœur de la première étape du cycle : l'étape de «structuration des problèmes» qui consiste à développer une vision à long terme et de formuler des objectifs de transition. L'arène de transition «doit être conçue comme une arène virtuelle ou un réseau qui procure un espace pour la réflexion à long terme et l'expéri-

41. Ibid.; Derk Loorbach, op. cit.

42. Derk Loorbach et Jan Rotmans, op. cit., p. 238. 
mentation prolongét ${ }^{43}{ }^{»}$. Cette arène comprendra entre 15 et 20 personnes choisies par l'équipe de recherche pour participer aux débats devant mener à la «coproduction" d'une compréhension commune des problèmes de durabilité et des solutions disponibles. Cette compréhension commune est d'ailleurs un «résultat clé» de l'arène de transition puisqu'elle inspirera les étapes subséquentes du cycle. Les chercheurs ayant élaboré cette méthode notent que la sélection des participants à l'arène de transition est d'une importance vitale pour le succès du cycle: l'ouverture d'esprit, la capacité de remettre en question ses propres analyses et d'accepter des compromis, ainsi que l'aptitude à s'approprier une vision à long terme sont considérées comme des préalables essentiels. Ces traits de personnalité permettront aux débats et négociations parfois tendus de mener à une vision commune qui formera le socle des prochaines étapes du cycle.

La deuxième phase est dite «tactique». Il s'agit de prolonger le travail de l'arène de transition dans un processus de développement d'un «agenda de transition", de réseautage et de coalition: l'objectif étant de rendre plus concrète la compréhension commune des problèmes et des solutions élaborées dans la phase précédente et de la partager avec un plus grand groupe d'acteurs. La construction de l'agenda de transition consistera à décliner cette compréhension commune de différentes manières: par secteur d'activité (transport, alimentation, logement, etc.), en élaborant des trajectoires précises et des «images de transition", etc. Ces déclinaisons résulteront de l'implication de nouveaux groupes d'acteurs (des citoyens, des entrepreneurs, des décideurs, des animateurs communautaires) et d'activités coordonnées de prospective (forecasting) et de rétrospective (backcasting) qui consistent à imaginer un avenir collectif soutenable et les avenues qui pourraient y mener. Ainsi, alors que la vision commune élaborée dans l'arène de transition demeurera le "pilier» de la gestion de la transition, «l'agenda de transition sera la boussole pour les acteurs avant-gardistes [frontrunners] qui pourront s'y référer au cours de leur processus de recherche et d'apprentissage ${ }^{44}{ }$.

Le cycle de la gestion des transitions est donc incrémental, et l'agenda de transition représentera une sorte de panier d'idées dans lequel l'arène de transition, accompagnée de l'équipe de chercheurs, choisira quelques initiatives à expérimenter. Cette troisième phase est donc nommée "opérationnelle». Définie de manière large, une expérience de transition est un projet d'innovation comportant un défi sociétal et qui agira comme point de

\footnotetext{
43. Jan Rotmans et Derk Loorbach, «Towards a Better Understanding of Transitions and Their Governance. A Systemic and Reflexive Approach», dans John Grin, Jan Rotmans et Johan Schot (dir.), op. cit., p. 157.

44. Ibid., p. 159.
} 
départ à l'apprentissage orienté vers une transition ${ }^{45}$. Ces expérimentations - choisies en fonction de leur adéquation et de leur cohérence avec la vision commune - sont également conçues comme une manière d'enrôler dans le processus de plus en plus de citoyens et d'utilisateurs grâce à leur matérialité et leur visibilité. Ainsi, un système de transport alternatif, une initiative de verdissement ou encore de partage alimentaire, par exemples, auront à la fois pour fonction de rendre une communauté plus «durable» et de faire connaitre le processus de gestion des transitions.

La quatrième phase vise plus spécifiquement à évaluer les résultats des expérimentations et des autres étapes du cycle, notamment à l'égard des connaissances qu'elles génèrent et des apprentissages collectifs qu'elles promeuvent. La communication et l'apprentissage sont d'ailleurs au cœur du processus « réflexif» de la gestion des transitions, lequel est à la fois considéré comme le cœur de cette quatrième phase du cycle et comme une caractéristique transversale à l'ensemble des quatre phases. Ainsi, la transversalité de la pensée réflexive signifie que «l'intégration du contrôle et de l'évaluation au sein de chaque phase et à chaque niveau de la gestion des transitions peut stimuler un processus d'apprentissage émergent de l'interaction et de la coopération entre différents acteurs impliqués ${ }^{46}{ }^{»}$.

Les publications relatant les cas où le cycle de la gestion des transitions a entraîné des changements bénéfiques dans les communautés locales et les municipalités sont nombreuses. Une condition de réussite, qui est également un grand défi, est de rendre le processus continue, afin que l'engagement des chercheurs avec un groupe d'acteur se poursuive dans le temps et génère une dynamique à plus long terme. Ainsi, les promoteurs de la gestion des transitions affirment que le cycle devrait être repris plusieurs fois - dans l'ordre ou dans le désordre - tout en maintenant la continuité de l'arène de transition. On peut par exemple imaginer que suite à l'étape réflexive, l'équipe de l'arène de transition désire revenir sur l'agenda de transition ou encore mettre en place une nouvelle étape d'expérimentation.

D'autres chercheurs proposent également d'utiliser les principes généraux de la gestion de la transition sans toutefois tenter de reproduire le modèle du cycle qui paraitra trop rigide et inadapté à certains contextes de recherche et d'intervention ${ }^{47}$. Ces derniers font généralement valoir que la gestion des transitions doit avant tout être considérée comme un modèle de

45. $I d$.

46. Ibid., p. 160

47. Julia M. Wittmayer et Niko Schäpke, «Action, research and participation: roles of researchers in sustainability transitions», Sustainability Science, vol. 9, 2014, p. 483-496; René Audet et Marie-Françoise Guyonnaud, «Transition in Practice and Action in Research. A French Case Study in Piloting Eco-innovations», Innovation: The European Journal of Social Sciences, vol. 26, n 4, 2013, p. 398-415. 
recherche-action spécifique, orienté par les cadres théoriques de la réflexivité et de la perspective multiniveaux, mais qu'il faut l'adapter en fonction des projets et des contextes. D'ailleurs, au-delà de sa pertinence scientifique et méthodologique, ce dernier argument cache parfois un inconfort à l'endroit des pratiques de «branding» que certains des ténors du champ des sustainability transitions promeuvent afin de renforcer la présence de leurs concepts dans plusieurs espaces institutionnels - que ce soit au niveau politique en faisant la promotion de la vision sociotechnique de la transition ou au niveau universitaire en investissant des espaces stratégiques à haut «facteur d'impact». De plus, les débats très ouverts qui se tiennent lors des conférences annuelles et les petites controverses ponctuelles qui éclatent sur la liste d'envoi électronique du réseau ${ }^{48}$ témoignent d'une vigilance face au dogmatisme que des outils théoriques et méthodologiques peuvent engendrer s'ils ne sont pas soumis à un examen critique systématique. Il est tentant d'affirmer, d'ailleurs, que cet examen critique a fait émerger un quatrième courant au sein du champ des sustainability transitions, qui est celui de la critique épistémologique et politique. En guise de conclusion, j'aborderai quelques enjeux soulevés par cette critique.

\section{La critique épistémologique et politique des sustainability transitions}

J'ai montré que la convergence intellectuelle qui est à l'origine du champ des sustainability transitions reposait sur des concepts - la réflexivité et la complexité - engendrés par des traditions scientifiques distinctes, mais qui avaient en commun de s'intéresser au changement dans le cadre de la crise écologique. Dans la dynamique concrète du champ, bien sûr, les chercheurs penchent généralement davantage du côté de la réflexivité s'ils proviennent des sciences sociales, et davantage du côté de la complexité s'ils proviennent des sciences de l'environnement. Mais il demeure néanmoins que le champ fait preuve d'une cohésion certaine en proposant une cohabitation des deux concepts. Ainsi, même les sociologues promoteurs de la perspective multiniveaux n'hésitent pas à véhiculer des métaphores naturalistes comme la «niche», le "paysage» ou la «sélection», alors que les tenants des systèmes complexes insistent sur l'équivalence des deux concepts lorsqu'il s'agit de proposer des recommandations pour le pilotage du changement ${ }^{49}$. Cette cohabitation des concepts a clairement une fonction rassembleuse et structurante pour le champ. Toutefois, il me semble exister une incohérence au

48. Observations personnelles de l'auteur.

49. Voir Jan Rotmans et Derk Loorbach, op. cit, p. 144-160. 
niveau de la traduction de ces concepts dans les cadres d'analyse des sustainability transitions - j'entends par là que la réflexivité et la complexité ne sont pas comprises de la même manière par les deux filiations.

Pour les sociologues, la réflexivité est une compétence sociale qui suppose la capacité, pour les acteurs sociaux, de se représenter et de décrire leurs pratiques dans le contexte de leurs conditions sociales. C'est en quelque sorte ce que Giddens appelle la $"$ conscience discursive $»^{50}$. Chez Beck, cette même réflexivité est pensée comme un attribut de plus en plus stratégique pour évoluer en tant qu'acteur social dans la société du risque, et aussi pour y trouver des modes de gouvernance les plus appropriés possibles ${ }^{51}$. Pour les deux sociologues, cette réflexivité est stratégique parce que les problèmes sont de plus en plus complexes, au sens plus générique de «non appréhendable dans leur entièreté ${ }^{52}$. Or, chez les promoteurs du concept de complexité, la réflexivité est toute autre chose: il s'agit d'une réflexion sur les principes théoriques de la complexité, au sens théorique développé par la science des systèmes complexes. Celle-ci constitue avant tout une compétence "émergente» d'un processus «d'auto-organisation» - c'est-à-dire l'organisation spontanée d'un système ${ }^{53}$. En somme, les concepts de réflexivité et de complexité tels que pensés par les deux filiations sont à la fois différents et analogues, ce qui engendre des glissements sémantiques et, surtout, une logique de justification qui est circulaire et tautologique : la complexité nécessite une plus grande réflexivité qui engendre de la complexité. Mais à chaque itération, parle-t-on toujours de la même réflexivité et de la même complexité?

Du point de vue de la sociologie de l'environnement, qui étudie les relations entre sociétés et environnement, il s'agit là d'un enjeu important. Depuis que les premières conceptualisations «écologiques» de ces relations ont donné lieu à des réductionnismes parfois moralement regrettables ${ }^{54}$, l'usage des métaphores naturalistes est suspect. Le cas de la théorie des systèmes complexes est d'autant plus singulier qu'il s'agit d'une théorie largement bâtie sur la physique thermodynamique et les mathématiques, et dont le principe de production de connaissance est avant tout la projection fondée sur des modélisations. Au champ des sustainability transitions, le sociologue de l'environnement posera donc la question suivante: étant donné, d'une

50. Anthony Giddens, op. cit.

51. Ulrich Beck, World at Risk, op. cit.

52. Urlich Beck, Anthony Giddens et Scott Lash, Reflexive Modernisation. Politics, Tradition and Aesthetics in the Modern Social order, Stanford, Stanford University Press, 1994.

53. Jan Rotmans et Derk Loorbach, «Towards...», op. cit., Jan Rotmans et Derk Loorbac, «Complexity... », op. cit.

54. Je pense notamment aux idées de la première écologie humaine de l'école de Chicago qui a pu traité les enjeux de discrimination raciale comme des phénomènes écologiques «naturels». Voir: Rodrick D. McKensie, «L'approche écologique dans l'étude de la communauté humaine», dans Yves Grafmeyer et Isaac Joseph, L'École de Chicago: naissance de l'écologie urbaine, Paris, Éditions du Champ urbain, 1979, p. 149-166. 
part, la justification tautologique des paradigmes de la complexité et de la réflexivité et, d'autre part, l'aspect métaphorique du paradigme de la complexité, ne vaut-il pas mieux abandonner ce dernier pour recentrer l'analyse autour du paradigme de la réflexivité?

Ce débat épistémologique est ouvert et se déroule au sein même du champ, où il côtoie un autre débat exposant des critiques plus disparates, mais qui concernent toutes la dimension politique des sustainability transitions. Il semble que les principaux auteurs du champ aient très tôt appréhendé ces critiques: en 2006, par exemple, Kemp et Loorbach formulaient cette mystérieuse mise en garde concernant leur cadre de pilotage du changement: «il devrait être clair que la gestion des transitions n'est pas une tentative mégalomaniaque pour contrôler le futur ${ }^{55} \ldots »$. Ainsi, il était déjà clair que le champ était exposé aux critiques politiques, mais il a fallu un certain temps avant que les positions défensives ne donnent place à des réponses plus constructives, ce qui fait que les débats actuels sur la dimension politique des sustainability transitions évoluent rapidement. J'essaie ici d'en faire un compte rendu en trois points.

Une première critique s'attarde à l'aspect fortement technocentriste de la vision du changement que proposent les approches des sustainability transitions. Cela est peut-être encore plus visible avec la perspective multiniveaux et l'amalgame qu'on y trouve entre innovation et innovation sociotechnique. Or, il existe actuellement un grand nombre de mouvements citoyens, d'initiatives locales et de courants d'analyse théoriques qui mettent davantage l'accent sur le rôle de l'innovation sociale tout en se réclamant aussi de la transition écologique ${ }^{56}$. Le peu d'intérêt accordé aux modèles plus subversifs de changement social dans la perspective multiniveaux révèle un préjugé favorable aux facteurs technologiques dans l'explication du changement social. On peut d'ailleurs légitimement se demander jusqu'à quel point le champ des sustainability transitions réussit véritablement à sortir du paradigme de la modernisation écologique, dont les limites ont été décrites dans la première partie de cet article. Le caractère politique de cette critique n'apparaît peut-être pas encore immédiatement, mais il devient nettement mieux exposé à la lumière des deux arguments suivants.

Un deuxième groupe de critiques dénonce le caractère apolitique du champ des sustainability transitions, c'est-à-dire la tendance à occulter les

55. René Kemp et Derk Loorbach, op. cit., p. 126.

56. Voir: Andrian Smith et Adrian Ely, «Green transformation from below? The politics of green transformation», dans Ian Scoones, Melissa Leach et Peter Newell (dir.), The Politics of Green Transformation, Londres et New York, Routledge, 2015, p. 102-118; Rob Hopkins, Ils changent le monde! 1001 initiatives de transition écologique, Paris, Seuil, 2014; voir aussi le texte de Chanez et Paré dans ce numéro. 
relations de pouvoir qui existent dans tout processus de changement social. Ces critiques prennent deux cibles. Les premières insistent surtout sur l'absence de questionnement à l'égard des rapports de pouvoir pouvant exister entre les acteurs prenant part à un processus de gestion des transitions ${ }^{57}$. Un certain nombre de questions sont évoquées pour illustrer cet angle mort du champ: la transition sera durable pour qui? Elle se fera avec qui? Qui en seront les exclus? Les réponses à ces questions demeurent encore imprécises aujourd'hui, mais comme je l'ai indiqué plus haut elles commencent à être explorées par les chercheurs qui considèrent que la gestion des transitions est un modèle parmi d'autres de recherche-action, et que les enjeux de pouvoir sont très directement posés dans ces autres traditions qui peuvent aussi contribuer à un pilotage des transitions ${ }^{58}$.

La deuxième cible à laquelle s'applique le reproche d'apolitisme est la perspective multiniveaux. Malgré l'importance qu'y trouvent les notions de règle et de verrouillage pour expliquer la résistance des régimes sociotechniques au changement, l'approche multiniveaux ne théorise pas des objets comme le conflit, le pouvoir ou encore le discours politique ${ }^{59}$. Frank Geels, qui fut au cœur du développement de la perspective multiniveaux, a récemment tenté une synthèse de son cadre théorique en y intégrant des principes d'économie politique et d'analyse du discours, mais la mise en œuvre de cette «réforme» dans des projets de recherche concrets demande encore à être constatée.

Au-delà de leur pertinence scientifique, les deux critiques précédentes prennent leur importance dans une troisième qui repose sur une analyse plus contextuelle du champ des sustainability transitions dans les débats contemporains entourant l'environnement et le développement. Ces débats font une place croissante à l'idée de transition à laquelle on accole un grand nombre de suffixes qui renvoient à des projets politiques variés et potentiellement contradictoires: transition vers l'économie verte ou vers l'économie sobre en carbone, transition énergétique ou transition écologique, transition juste, etc. C'est donc aussi dans ce contexte discursif plus large qu'il faut situer l'émergence du champ des sustainability transitions, ce qui implique de penser l'interaction entre champ académique et discours politique $^{60}$. Comme l'explique Giddens, les théories élaborées par les chercheurs

57. Elizabeth Shove et Gordon Walker, «CAUTION! Transitions ahead: politics, practice and sustainable transition management », Environment and Planning A, vol. 39, 2007, p. 763-770.

58. Julia M. Wittmayer et Niko Schäpke op. cit.; Julia Maria Wittmayer, Niko Schäpke et Frank van Steenbergen, op. cit.

59. James Meadowcroft, «Engaging with the Politics of Sustainability Transitions», Environmental Innovation and Societal Transitions, vol. 1, p. 70-75.

60. René Audet, «The Double Hermeneutic of Sustainability Transitions», Environmental Innovation and Societal Transitions, vol. 11, 2014, p. 46-49. 
en sciences sociales sont constamment réappropriées et réinterprétée par les acteurs sociaux qui transforment par le fait même la réalité que ces théories veulent décrire ${ }^{61}$. Il est évidemment difficile de mettre à jour toutes les ramifications que le champ des sustainability transitions a implanté dans le terreau du discours de la transition, mais sa proximité avec les institutions gouvernementales néerlandaises et l'influence qu'il a déjà sur les politiques environnementales dans certains pays européens indiquent que son impact est concret. La double herméneutique de la transition est donc bien réelle et elle fait en sorte que la vision technocentriste, souvent apolitique et parfois naturaliste, que véhicule le champ des sustainability transitions, influence la réalité qu'elle tente de décrire et de piloter, mais aussi les discours et les pratiques de nombreux acteurs qui, eux, sont engagés dans une démarche incontestablement politique. 